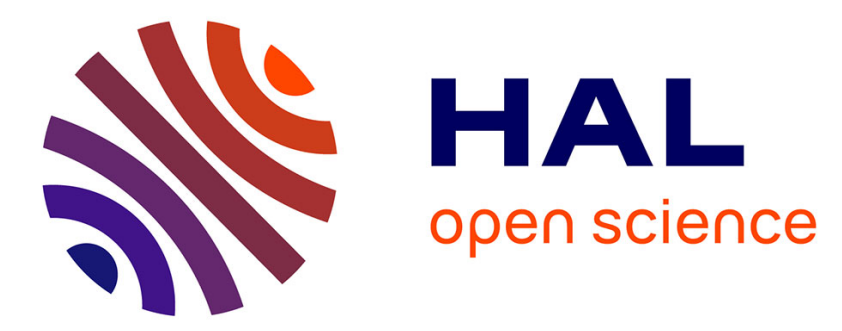

\title{
Numerical modeling of soil-pipe interaction of single pipeline at shallow embedment in clay by hypoplastic macroelement
}

\author{
Zhuang Jin, Zheng Li, Zhen-Yu Yin, Panagiotis Kotronis
}

\section{To cite this version:}

Zhuang Jin, Zheng Li, Zhen-Yu Yin, Panagiotis Kotronis. Numerical modeling of soil-pipe interaction of single pipeline at shallow embedment in clay by hypoplastic macroelement. Ocean Engineering, 2021, 241, pp.110017. 10.1016/j.oceaneng.2021.110017 . hal-03403693

\section{HAL Id: hal-03403693 \\ https://hal.science/hal-03403693}

Submitted on 27 Jan 2022

HAL is a multi-disciplinary open access archive for the deposit and dissemination of scientific research documents, whether they are published or not. The documents may come from teaching and research institutions in France or abroad, or from public or private research centers.
L'archive ouverte pluridisciplinaire HAL, est destinée au dépôt et à la diffusion de documents scientifiques de niveau recherche, publiés ou non, émanant des établissements d'enseignement et de recherche français ou étrangers, des laboratoires publics ou privés.

\section{(c) (1) $\$$}

Distributed under a Creative Commons Attribution - NonCommerciall 4.0 International 
Author Accepted Manuscript of the article published in Ocean Engineering, 241, 2021 https://doi.org/10.1016/j.oceaneng.2021.110017

\title{
Numerical modeling of soil-pipe interaction of single pipeline at shallow embedment in clay by hypoplastic macroelement
}

\author{
Zhuang JIN ${ }^{\mathrm{a}}$, Zheng LI ${ }^{\mathrm{b}, *}$, Zhen-yu YIN ${ }^{\mathrm{c}, * *}$, Panagiotis KOTRONIS
}

${ }^{a}$ Department of Ocean Science and Engineering, Southern University of Science and Technology, Shenzhen, China ${ }^{b}$ Université Gustave Eiffel, Department GERS, Laboratoire Centrifugeuses Géotechnique (CG), 44341 Bouguenais, France

${ }^{c}$ Department of Civil and Environmental Engineering, The Hong Kong Polytechnic University, Kowloon, Hong Kong, China ${ }^{d}$ École Centrale de Nantes, Université de Nantes, CNRS, Institut de Recherche en Génie Civil et Mécanique (GeM), UMR 6183, 1 rue de la No e, BP 92101, 44321, Nantes, cedex 3, France

\begin{abstract}
Nowadays, the numerical analysis of submarine pipelines of offshore oil and gas industry is a big challenge in engineering design. A simple, fast and accurate numerical tool is proposed in this article based on the macroelement concept. The novel macroelement is within the framework of hypoplasticity and can consider static monotonic combined (multi-directional) loads for shallow embedded pipelines in clay. The incremental nonlinear constitutive formulas are defined in terms of generalised forces and displacements and an enhanced function of failure surface is introduced. A series of empirical formulas are proposed to describe the stiffness variation trends for soil-pipeline interaction. Model predictions show that the proposed macroelement is proved to be an efficient alternative approach compared to the traditional finite element analysis. The computational cost is thus much reduced for the pipeline design.
\end{abstract}

Keywords: Pipeline; Clay; Soil-pipeline interaction; Hypoplasticity; Macroelement; Offshore engineering

\section{Introduction}

Pipelines are critical link among oil and gas field, offshore wind farms and related product users onshore. As oil and gas developments move into deeper water, the offshore pipelines represent an increasingly significant part of the facility costs. In deep water, pipelines are generally laid on the seabed, penetrating by a fraction of a diameter due to their own weight and the effects of the laying process. Submarine pipelines are subjected to high temperatures and pressures during service, causing axial expansion and subsequent lateral buckling of the pipelines [1]. The degree of buckling along the horizontal direction mainly depends on the lateral soil resistance. It is worthy noting that the sweeping of pipeline due to buckling across the seabed could neutralize partial axial loadings. At the same time, excessive buckling causes stress concentration in the pipeline, which has an irreversible negative effect on the service life of the pipeline. For design purposes, predicting the stability behavior and understanding the performance of pipelines under combined environmental loadings is therefore of great importance.

The numerical simulation is widely adopted to analyse the nonlinear behavior of pipeline [2-4]. A great number of large deformation based finite element analyses were carried out and the effect of strain rate and softening effects are highlighted [5-10]. However, the nonlinear finite element analyses are usually time-consuming and require considerable skills. An alternative high-efficient and convenient practical approach to reproduce the nonlinear behaviour of foundations under combined loadings is the so-called macroelement approach introduced in geotechnical engineering by Nova and Montrasio [11]. According to the concept of macroelement, the nonlinear behavior of the soil-structure system is modeled by relating the resultant forces directly to the corresponding displacement through a reference point [12].

The early applications of the macroelement tool were for the foundations under monotonic and cyclic/dynamic loading conditions [12-29]. The macroelement models mentioned above were developed with the conventional

\footnotetext{
${ }^{*}$ Corresponding author

${ }^{* *}$ Co-corresponding author

Email address: zheng.li@univ-eiffel.fr; lizheng619@hotmail .com (Zheng LI)
} 
$$
30
$$

43 finite element simulations (FEM).

\section{2. Problem definition}

The problem studied in this paper is concisely sketched in Fig. 1. A shallow embedded pipe is considered ${ }_{46}$ resting on an infinite clay strata. The pipe has a diameter $D$ with an embedment depth $w$. $w / D$ ranges from 0.1 47 to 0.5 , which is the zone of most interest for typical single-bore pipelines [2]. The undrained shear strength of 48 clay is $s_{u}$. A representative segment $L$ in the longitude direction of the pipeline is chosen for the analysis. For 49 a plane strain problem, the length $L$ is taken as the unit length of the unit system. $u$ and $w$ are the horizontal ${ }_{50}$ and vertical displacements at the center of pipe. $H$ and $V$ are the corresponding horizontal and vertical forces 51 applied on. Two extreme contact conditions i.e. frictionless and rough are considered in this study. A parameter ${ }_{52} \alpha$ is used to describe these two contact conditions. For frictionless condition $\alpha=0$ while $\alpha=1$ for rough 53 condition [2].

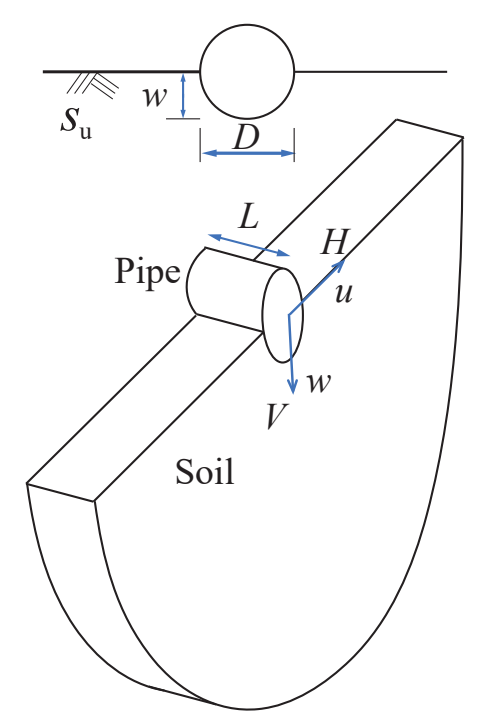

Figure 1: Sketch of soil-pipe system 


\section{3. Modeling of soil-pipe interaction by hypoplastic macroelement: constitutive framework}

In the framework of hypoplastic macroelement, the constitutive equation is established between the gener-

56 alized load vector $\boldsymbol{t}$ and the generalized displacement vector $\boldsymbol{d}$. For the soil-pipline interaction the moment can

57 be neglected and therefore the generalized load vector components are $\boldsymbol{t}=\left[\begin{array}{ll}V & H\end{array}\right]^{T}$ and $\boldsymbol{d}=\left[\begin{array}{ll}w & u\end{array}\right]^{T}\left(\begin{array}{l}w \\ \mathrm{and}\end{array} u\right.$ are

58 the pipe vertical displacement and lateral movement respectively), see Fig. 1. The constitutive equation for a

59 hypoplastic macroelement is established in rate-form $[33,36]$, which enables the consideration of non-linear

60 and irreversible behavior, Eqs. (1) and (2).

$$
\dot{\boldsymbol{t}}=\mathcal{K}(\boldsymbol{t}, \boldsymbol{\eta}) \dot{\boldsymbol{d}}
$$

$$
\mathcal{K}=\mathcal{L}(\boldsymbol{t})+N(\boldsymbol{t}) \boldsymbol{\eta}^{T} \quad \boldsymbol{\eta}:=\frac{\dot{d}}{\|\dot{d}\|}
$$

${ }_{61}$ where $\dot{\boldsymbol{t}}$ and $\dot{\boldsymbol{d}}$ are respectively the generalized force rate and stretching velocity; $\mathcal{L}$ and $\boldsymbol{N}$ are constitutive 62 functions which depend on the current load state. In eq. $(2)^{1}$, the first term, $\mathcal{L}(\boldsymbol{t})$, on the right-hand side 63 represents the incrementally linear response of the constitutive equation. The second term, $N(t) \eta^{T}$, nonlinear 64 in $\dot{\boldsymbol{d}}$, is responsible for the incremental non-linearity of the system response [33]. $\boldsymbol{\eta}$ is the direction of the 65 stretching velocity.

To adapt the model to cyclic loading the model for cyclic loading, an additional internal variable namely "internal displacement" $\delta$ is introduced [45]. The internal displacement state variable is included with the following evolution equation:

$$
\begin{aligned}
& \dot{\boldsymbol{\delta}}=\widehat{\mathcal{H}}(\boldsymbol{\delta}, \boldsymbol{\eta}) \dot{\boldsymbol{d}} \quad \widehat{\mathcal{H}}= \begin{cases}\mathcal{I}-\rho^{\beta_{r}} \boldsymbol{\eta}_{\delta} \boldsymbol{\eta}_{\delta}^{T} & \text { if } \boldsymbol{\eta}_{\delta} \cdot \boldsymbol{\eta}>0 \\
\mathcal{I} & \text { if } \boldsymbol{\eta}_{\delta} \cdot \boldsymbol{\eta} \leq 0 .\end{cases} \\
& \boldsymbol{\eta}_{\delta}:=\left\{\begin{array}{ll}
\boldsymbol{\delta} /\|\boldsymbol{\delta}\| & (\text { if }\|\boldsymbol{\delta}\|>0) \\
\mathbf{0} & (\text { if }\|\boldsymbol{\delta}\|=0)
\end{array} \quad \rho:=\frac{1}{R}\|\boldsymbol{\delta}\|\right.
\end{aligned}
$$

${ }_{66}$ where $\mathcal{I}$ is an identity matrix; $\beta_{r}$ and $R$ are model constants; $\boldsymbol{\eta}_{\delta}$ provides the direction of $\boldsymbol{\delta} ; \rho \in[0,1]$ is a normalized measure of the internal displacement magnitude.

With the incorporation of the additional state variable [33,45], the constitutive equations of the macroele69 ment become eq. (5):

$$
\dot{\boldsymbol{t}}=\widehat{\mathcal{K}}(\boldsymbol{t}, \delta, \eta) \dot{d}
$$

where:

$$
\begin{gathered}
\widehat{\mathcal{K}}=\left[\rho^{\chi} m_{T}+\left(1-\rho^{\chi}\right) m_{R}\right] \mathcal{L}(\boldsymbol{t})+\widetilde{\mathcal{K}}(\boldsymbol{t}, \boldsymbol{\delta}, \boldsymbol{\eta}) \\
\widetilde{\mathcal{K}}= \begin{cases}\rho^{\chi}\left(1-m_{T}\right)\left(\mathcal{L} \boldsymbol{\eta}_{\delta}\right) \boldsymbol{\eta}_{\delta}^{T}+\rho^{\chi} \boldsymbol{N} \boldsymbol{\eta}_{\delta}^{T} & \text { (if } \left.\boldsymbol{\eta}_{\delta} \cdot \boldsymbol{\eta}>0\right) \\
\rho^{\chi}\left(m_{R}-m_{T}\right)\left(\mathcal{L} \boldsymbol{\eta}_{\delta}\right) \boldsymbol{\eta}_{\delta}^{T} & \text { (if } \left.\boldsymbol{\eta}_{\delta} \cdot \boldsymbol{\eta} \leq 0\right)\end{cases}
\end{gathered}
$$

70 where $\chi, m_{T}$ and $m_{R}$ are model constants.

Eqs. (3) to (7) imply that the tangential responses of the hypoplastic macrolement vary according to the development of the "internal displacement" $\boldsymbol{\delta}$. The "internal displacement" controls $\rho$ and $\boldsymbol{\eta}_{\delta}$ which record the history of the previous loading step. The term $\boldsymbol{\eta} \cdot \boldsymbol{\eta}_{\delta}$ determines whether the current loading is continues, reverses or is neutral (where the loading is tangential to the yield surface) compared to the previous loading step. The 75 magnitude of $\rho$ determines whether the current loading is far from the loading initiation or loading reversal. In this way the performance of the hypoplastic macroelement under cyclic loading is improved [32, 33, 45].

In this section, the general framework of the hypoplasticity macroelement is presented. However, several 78 key ingredients have to be reformulated to adapt the macroelement for soil-pipe interaction: the initial elastic 79 linear behavior defined by $\mathcal{L}$ and the development of the nonlinear behavior defined by $\boldsymbol{N}$. Furthermore, the so related parameters should be calibrated. These issues are presented in the following section. 


\section{Definitions of key ingredients of the constitutive relationship}

82 4.1. Elasticity

83 In hypolasticity, the tangent stiffness $\widehat{\mathcal{K}}$ varies continuously with the direction $\boldsymbol{\eta}$ of the generalized velocity

${ }_{84} \dot{\boldsymbol{d}}$. With the development of plasticity, the initial elasticity vanishes when the stretching $\boldsymbol{d}$ is sufficient large. The

85 initial elasticity is defined by a stiffness matrix $\mathcal{L}$ :

$$
\mathcal{L}:=\frac{1}{m_{R}}\left[\begin{array}{cc}
k_{\mathrm{vv}} & k_{\mathrm{hv}} \\
0 & k_{\mathrm{hh}}
\end{array}\right]
$$

${ }_{86}$ where $k_{\mathrm{vv}}, k_{\mathrm{hh}}$ and $k_{\mathrm{hv}}$ and are the vertical, horizontal and coupled stiffness coefficients of the soil-pipe system.

${ }_{87}$ The stiffness matrix $\mathcal{L}$ is asymmetric as there exists only one coupled stiffness $k_{\mathrm{hv}}$. The coupled $k_{\mathrm{hv}}$ is due to

88 the fact that for the soil-pipe interaction problem, the vertical downward displacement $w$ with the constraint of

89 lateral movement $(u=0)$ causes only a vertical reaction force. However, when the pipe is subjected to horizontal

9o displacement $u$ with the constraint of vertical movement $(w=0)$, both horizontal and vertical reaction forces

${ }_{91}$ exist. In accordance with the components of $\boldsymbol{d}=\left[\begin{array}{ll}w & u\end{array}\right]^{T}$, the upper right component of $\mathcal{L}$ is placed with the

92 coupling stiffness $k_{\mathrm{hv}}$. To quantify the stiffness components of the soil-pipe system, numerical simulations are

${ }_{93}$ carried out in this article. Fig. 2 shows the FEM mesh and boundary conditions of the numerical model. The size

94 of the soil domain is $6 \times 16 \mathrm{~m}$. The displacements at the bottom of the model are fixed in the $X$ and $Y$ directions

95 and on the lateral sides in the normal direction. Elastic properties are assigned for soil with a Poisson's ratio

${ }_{96} v=0.49[2,46,47]$. The elastic modulus of the pipe is set to be 206GPa, so as to it can be regarded as a rigid

97 body. For the purpose of getting closer to the engineering practice, the variation of soil modulus with respect

98 to depth is considered following two profiles i.e. a constant modulus and a linear modulus profile, see Fig. 3 .

99 In this study, five values (40 MPa, $80 \mathrm{MPa}, 160 \mathrm{MPa}, 200 \mathrm{MPa}$ and $300 \mathrm{MPa}$ ) are set to cover the possible

100 soil modulus in practical pipe-line engineering. The constant modulus case represents an overconsolidated

101 clay, while the linear distribution a normally consolidated clay $[46,48]$. To determine numerically the stiffness

102 factors, small vertical and horizontal displacements are applied on the center of the pipe and the reaction forces 103 are measured. This method is illustrated in Fig. 4.

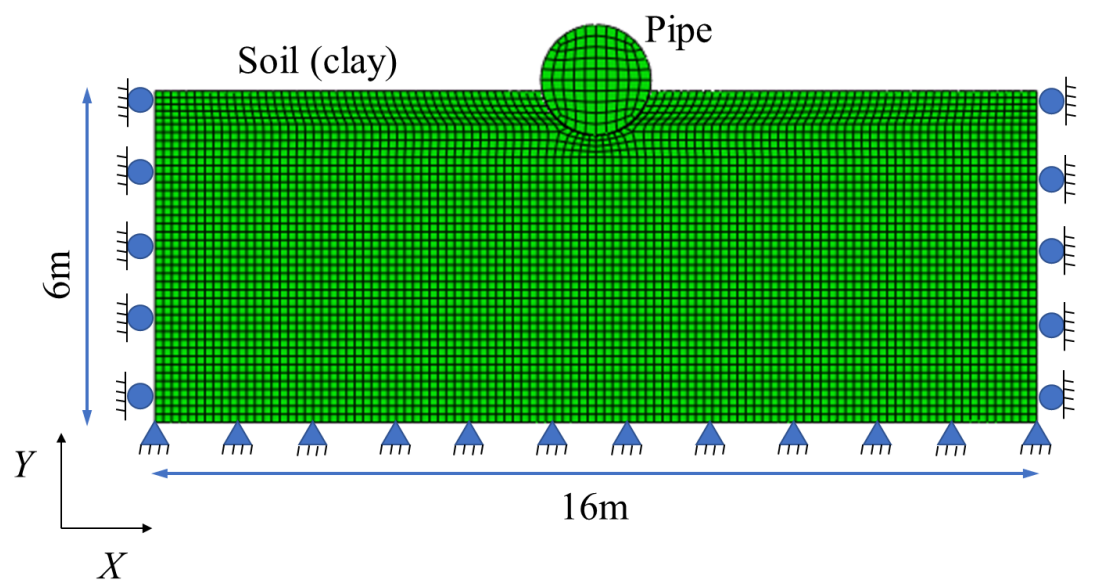

Figure 2: FEM mesh and boundary conditions 


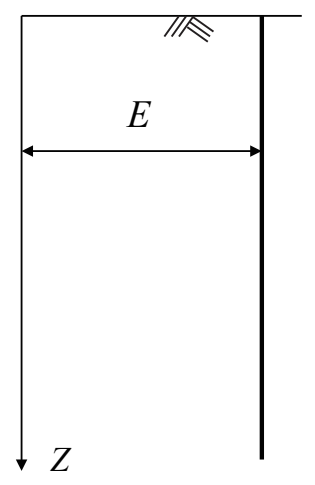

(a) Constant modulus profile

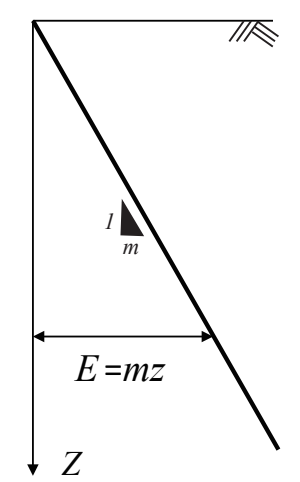

(b) Linear modulus profile

Figure 3: Soil stiffness profiles

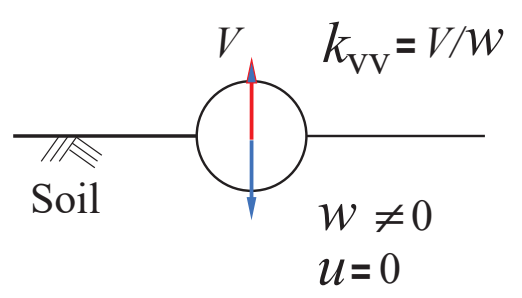

Figure 4: Determination of stiffness components

For a comprehensive study of the stiffness properties of soil-pipe system, parametric studies were carried out on the influence of pipe diameter, embedment depth, contact condition and soil profiles. Empirical equations are proposed to calculate the components in the stiffness matrix, see Tables. 1 and 2.

Table 1: Empirical equations for soil-pipe system with frictionless contact condition

\begin{tabular}{cccc}
\hline & Vertical stiffness $k_{\mathrm{vv}}$ & Horizontal stiffness $k_{\mathrm{hh}}$ & Coupled stiffness $k_{\mathrm{hv}}$ \\
\hline Constant profile & $0.28 E_{\mathrm{D}} D c_{\mathrm{D}}\left(1+1.45\left(\frac{w}{D}\right)^{0.22}\right)$ & $0.49 E_{\mathrm{D}} D c_{\mathrm{D}}\left(\frac{w}{D}\right)^{0.75}$ & $0.21 E_{\mathrm{D}} D c_{\mathrm{D}}\left(\frac{w}{D}\right)^{0.21}$ \\
Linear profile & $0.10 E_{\mathrm{D}} D c_{\mathrm{D}}\left(1+8.0\left(\frac{w}{D}\right)^{0.5}\right)$ & $0.36 E_{\mathrm{D}} D c_{\mathrm{D}}\left(\frac{w}{D}\right)^{0.96}$ & $0.23 E_{\mathrm{D}} D c_{\mathrm{D}}\left(\frac{w}{D}\right)^{0.55}$ \\
\hline
\end{tabular}

Table 2: Empirical equations for soil-pipe system with rough contact condition

\begin{tabular}{cccc}
\hline & Vertical stiffness $k_{\mathrm{vv}}$ & Horizontal stiffness $k_{\mathrm{hh}}$ & Coupled stiffness $k_{\mathrm{hv}}$ \\
\hline Constant profile & $0.26 E_{\mathrm{D}} D c_{\mathrm{D}}\left(1+3.0\left(\frac{w}{D}\right)^{0.42}\right)$ & $0.51 E_{\mathrm{D}} D c_{\mathrm{D}}\left(\frac{w}{D}\right)^{0.25}$ & $0.12 E_{\mathrm{D}} D c_{\mathrm{D}}\left(\frac{w}{D}\right)^{0.57}$ \\
Linear profile & $0.15 E_{\mathrm{D}} D c_{\mathrm{D}}\left(1+8.3\left(\frac{w}{D}\right)^{0.65}\right)$ & $0.49 E_{\mathrm{D}} D c_{\mathrm{D}}\left(\frac{w}{D}\right)^{0.66}$ & $0.13 E_{\mathrm{D}} D c_{\mathrm{D}}\left(\frac{w}{D}\right)^{0.8}$ \\
\hline
\end{tabular}

${ }_{114}$ frictionless/rough pipe-clay contact interface with linear/constant modulus profiles is presented in Figs. 5 and

115 Fig. 6. The proposed empirical equations have a good agreement with the FEM results. 


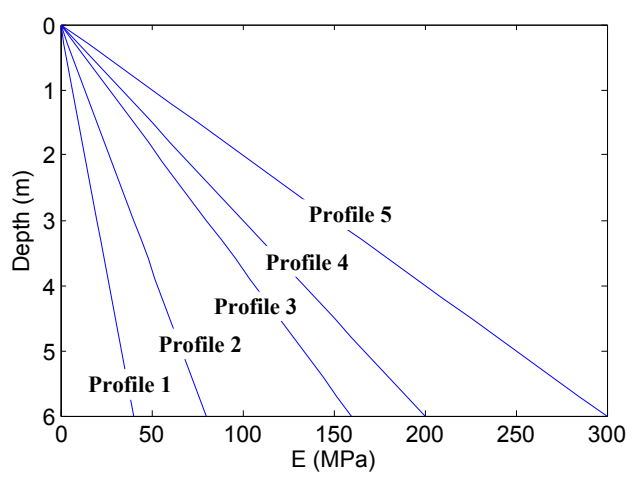

(a) Modulus profiles

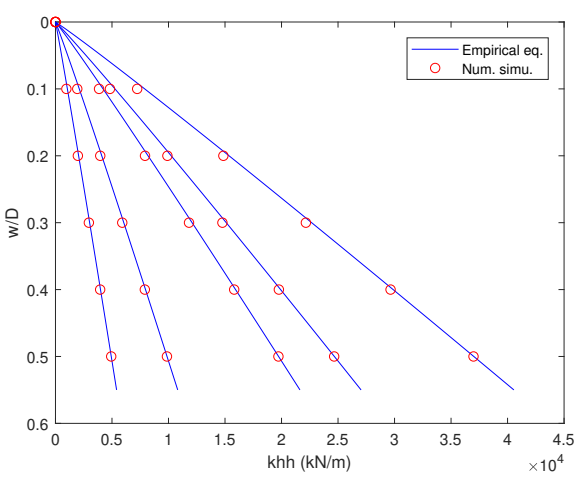

(c) Horizontal stiffness $k_{\mathrm{hh}}$

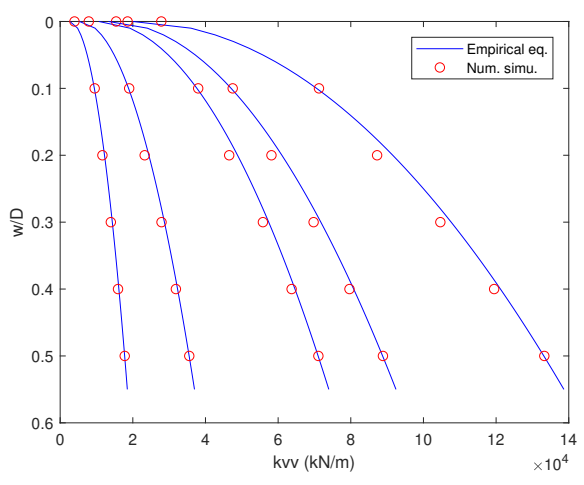

(b) Vertical stiffness $k_{\mathrm{Vv}}$

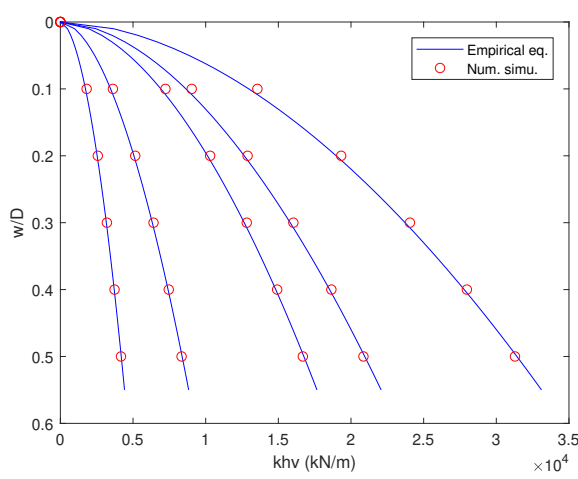

(d) Coupled stiffness $k_{\mathrm{hv}}$

Figure 5: Validation of empirical equations: frictionless contact (a) Young's modulus $E$ profiles - linear type (b) vertical elastic stiffness $k_{\mathrm{vv}}$ (c) horizontal elastic stiffness $k_{\mathrm{hh}}$ (d) coupled elastic stiffness $k_{\mathrm{hv}}$

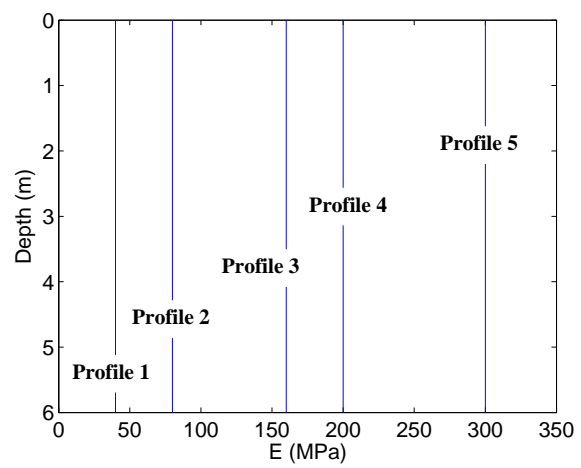

(a) Modulus profiles

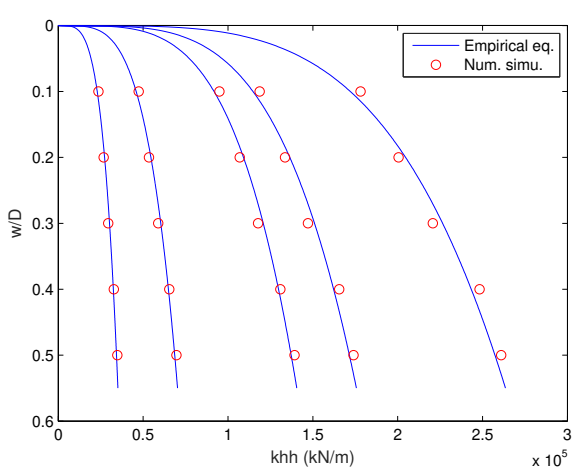

(c) Horizontal stiffness $k_{\mathrm{hh}}$

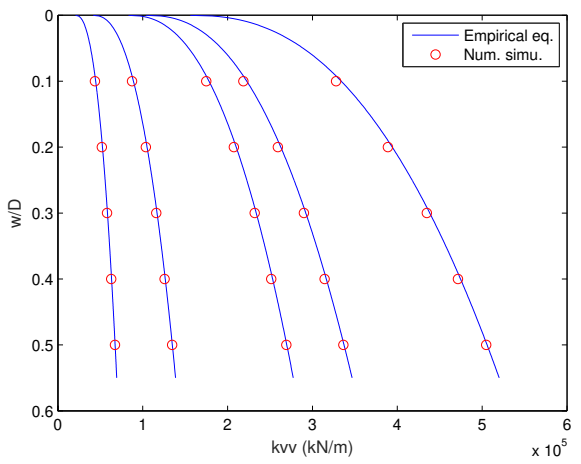

(b) Vertical stiffness $k_{\mathrm{vv}}$

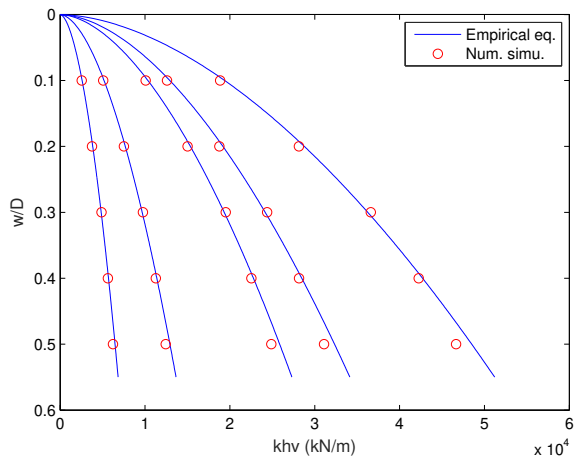

(d) Coupled stiffness $k_{\mathrm{hv}}$

Figure 6: Validation of empirical equations: rough contact (a) Young's modulus $E$ profiles - constant type (b) vertical elastic stiffness $k_{\mathrm{vy}}$ (c) horizontal elastic stiffness $k_{\mathrm{hh}}(\mathrm{d})$ coupled elastic stiffness $k_{\mathrm{hv}}$

The neccessary information for calculating the vertical stiffness of the pipepline are availble in some exsiting 117 studies [42, 49-53]. In order to further validate the empirical equations for soil-pipe systems, the related data 118 of several centrifuge tests (Dingle et al. [49]; Cheuk and White [50]; White and Dingle [51]) and model tests 
(Al-Janabi et al. [53]) are adopted to calculated the vertical and horizontal stiffness. The vertical stiffness is obtained by measuring the initial slope of the penetration tests i.e., the vertical load-embedment profiles presented in these researches. The horizontal stiffness can be also calculated with a similar procedure using lateral load-displacement response curves. Note that the penetration tests start from mudline, in other words, the initial embedment $w / D$ for the vertical stiffness calculation equals to 0 . The rough contact assumptions are adopted for all the model tests based on the descriptions in the references. Furthermore, the Young's modulus profile is considered linear according to the shear strength $s_{u}$ provided in these researches and the modulus ratio $\left(E / s_{u}\right)$ equals to 500 . The model parameters and soil properties for the stiffness terms calculations are summarized in Tables. 3 and 4.

Table 3: Experimental data used for validation of vertical stiffness

\begin{tabular}{|c|c|c|c|c|}
\hline Model test & $\begin{array}{l}\text { Pipe diameter } \\
\qquad D(\mathrm{~m})\end{array}$ & $\begin{array}{l}\text { Initial embedment } \\
\qquad w / D\end{array}$ & $\begin{array}{l}\text { Undrained shear strength } \\
\qquad s_{u}(\mathrm{kPa})\end{array}$ & Test No./type \\
\hline Centrifuge test, Cheuk and White [50] & 0.8 & 0 & $0.75+1.6 z$ & Test KC05 \\
\hline Centrifuge test, Hodder and Cassidy [42] & 0.5 & 0.5 & $3.5+0.7 z$ & Test NO. 1.305 .2 \\
\hline Model test & $\begin{array}{l}\text { Pipe diameter } \\
\qquad D(\mathrm{~m})\end{array}$ & $\begin{array}{l}\text { Initial embedment } \\
\qquad w / D\end{array}$ & $\begin{array}{l}\text { Undrained shear strength } \\
\qquad s_{u}(\mathrm{kPa})\end{array}$ & Test No./type \\
\hline Centrifuge test, White and Dingle [51] & 0.8 & 0.18 & $2.3+3.6 z$ & Test L4 \\
\hline
\end{tabular}

Figs. 7 (a) and (b) show the comparison between the empirical equations and the test results for vertical respectively. The ratio of the experimental values to the predicted values from the proposed empirical formula is also indicated as a percentage in the figure. The results from the empirical equations for calculating vertical and 


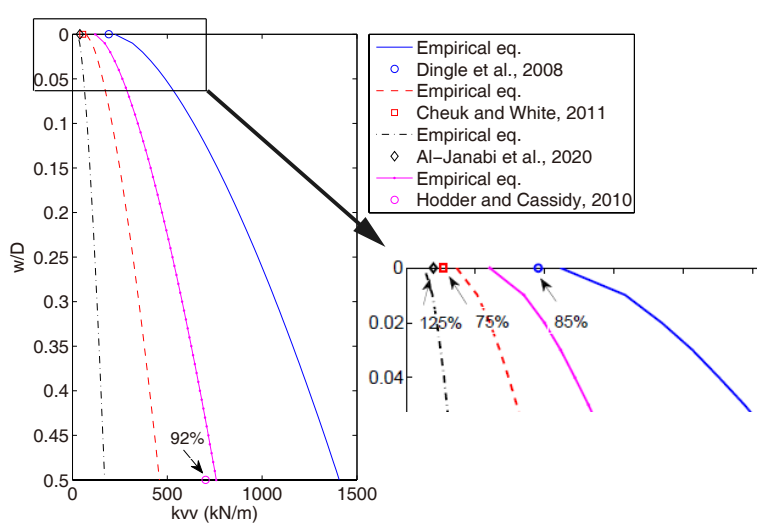

(a)

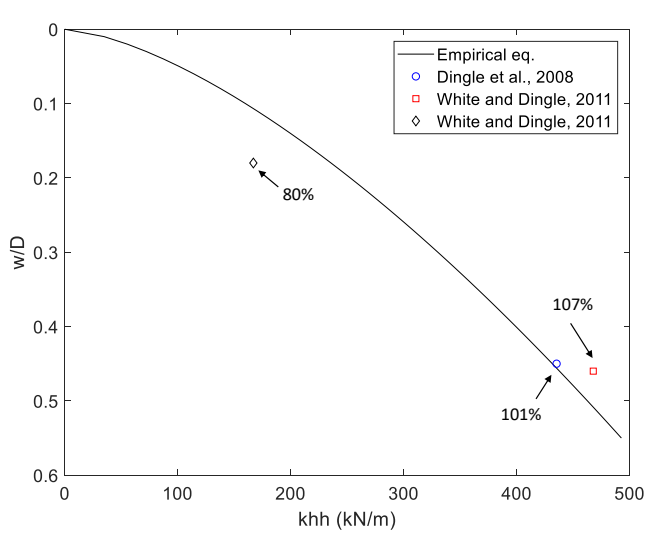

(b)

Figure 7: Validation of empirical equations for rough contact conditions with model test results: (a) vertical stiffness $k_{\mathrm{vv}}$ (b) horizontal stiffness $k_{\mathrm{hh}}$

\subsection{Plasticity}

\section{4.2.1. Bearing capacity envelope}

Merifield et al. [2] proposed a bearing capacity envelope with parabolic shape, which is adopted as the 136 bounding surface in the constitutive relationship of hypoplastic macroelement. In the framework of hypoplasticity, the envelope acts as a tractor to which the evolution of plasticity is referred. The envelope $f(\boldsymbol{t})=0$ is

138 defined as:

$$
f(\boldsymbol{t}):=\frac{H}{H_{\max }}-\beta\left(\frac{V}{V_{\max }}\right)^{\beta_{1}}\left(1-\frac{V}{V_{\max }}\right)^{\beta_{2}}=0
$$

where:

$$
\beta:=\frac{\left(\beta_{1}+\beta_{2}\right)^{\beta_{1}+\beta_{2}}}{\beta_{1}^{\beta_{1}} \beta_{2}^{\beta_{2}}} ; \quad H_{\max }:=V_{\max }\left(0.48-\frac{\alpha}{25}\right)\left(\frac{w}{D}\right)^{0.46-\frac{\alpha}{25}} ; \quad V_{\max }:= \begin{cases}7.4 s_{\mathrm{u}} D\left(\frac{w}{D}\right)^{0.4} & \text { (rough) } \\ 5.66 s_{\mathrm{u}} D\left(\frac{w}{D}\right)^{0.32} & \text { (frictionless) }\end{cases}
$$

139 $\beta_{1}$ and $\beta_{2}$ are two parameters defining the trend of varying skew with respect to embedment level: $\beta_{1}=(0.8-$ $0.15 \alpha)(1.2-w / D) ; \beta_{2}=0.35(2.5-w / D) . V_{\max }$ and $H_{\max }$ are the maximum vertical and horizontal resistance,

${ }_{141}$ respectively. The roughness parameter $\alpha$ takes values of 0 and 1 for frictionless and rough contact conditions, 142 respectively. $s_{u}$ is the undrained shear strength of clay.

143 4.2.2. Plasticity evolution

${ }_{144}$ As mentioned in section. 3, the constitutive function $N$ is responsible for the incremental non-linearity of 145 the system response. Proposed by Niemunis [32], the constitutive vector $\boldsymbol{N}$ of eq. (2) can be written as:

$$
N(t)=-Y(t) \mathcal{L} m(t)
$$

${ }_{146}$ where $Y(t) \in(0,1]$ is a scalar function which controls the degree of nonlinearity and increases with respect to

${ }_{147}$ the distance of the current stress state $\boldsymbol{t}$ to the ultimate bearing capacity envelope. $\boldsymbol{m}$ is a unit vector pointing 
148 the direction of the evolution of plasticity. The loading function $Y$ is defined by a simple power law:

$$
Y(\boldsymbol{t})=\xi^{k}
$$

${ }_{149}$ where $\xi$ is a nonlinear factor which measures the distance of the current loading surface to the bounding surface.

${ }_{150} \kappa$ is a model constant controlling the plastic hardening of the model response. For an arbitrary loading state $\boldsymbol{t}^{*}$

151 i.e. $\boldsymbol{t}^{*}=\left[\begin{array}{ll}V^{*} & H^{*}\end{array}\right]^{T}$ within the bounding envelope, there exists a scalar multiplier $\xi \in(0,1]$ which satisfies

${ }_{152} f\left(\boldsymbol{t}^{*}\right)=0$. The current loading state $\boldsymbol{t}^{*}$, the loading surface $f\left(\boldsymbol{t}^{*}\right)$ and bounding surface $f(\boldsymbol{t})$ are illustrated in

$153 \quad$ Fig. 9.

$$
f\left(\boldsymbol{t}^{*}\right):=\frac{H^{*}}{\xi H_{\max }}-\beta\left(\frac{V^{*}}{\xi V_{\max }}\right)^{\beta_{1}}\left(1-\frac{V^{*}}{\xi V_{\max }}\right)^{\beta_{2}}=0
$$

${ }_{154}$ After substituting the current loading state $\boldsymbol{t}^{*}=\left[\begin{array}{ll}V^{*} & H^{*}\end{array}\right]^{T}$ into eq. (13), the scalar multiplier $\xi \in(0,1]$ of the

155 current loading state can be obtained by solving the nonlinear function numerically by the Newton-Raphson

${ }_{156}$ method. In eq. $12, \kappa$ controls the plastic hardening in the model, which can be easily calibrated by comparing

157 the monotonic response with a given reference.

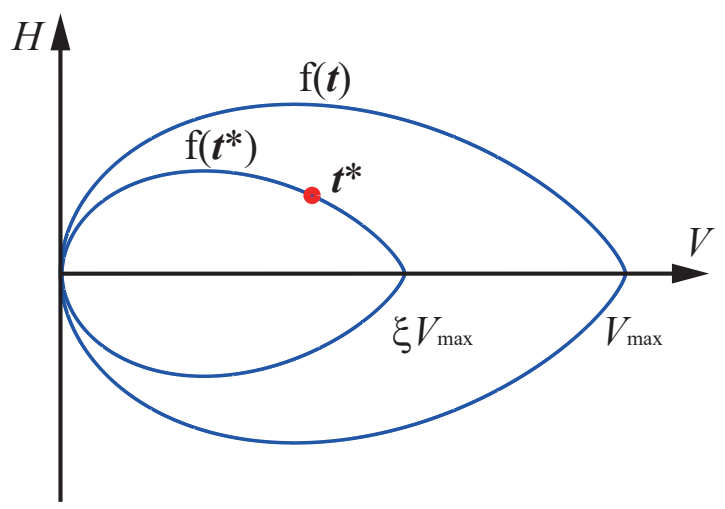

Figure 8: Determine the nonlinear factor $\xi$ from the current loading surface

158 The plastic flow direction $\boldsymbol{m}$ is defined by another function $g\left(\boldsymbol{t}_{g}\right)$ which differs from the loading and bounding 159 surface functions. A non-associated plastic potential function is chosen as the following:

$$
g\left(\boldsymbol{t}_{g}\right):=\frac{H_{g}}{\lambda_{h} \xi H_{\max }}-\left(\frac{1}{\lambda_{v}}\right) \beta\left(\frac{V_{g}}{\xi V_{\max }}\right)^{\beta_{1}}\left(1-\frac{V_{g}}{\xi V_{\max }}\right)^{\beta_{2}}=0
$$

160 where $\lambda_{h}$ and $\lambda_{v}$ are model parameters.

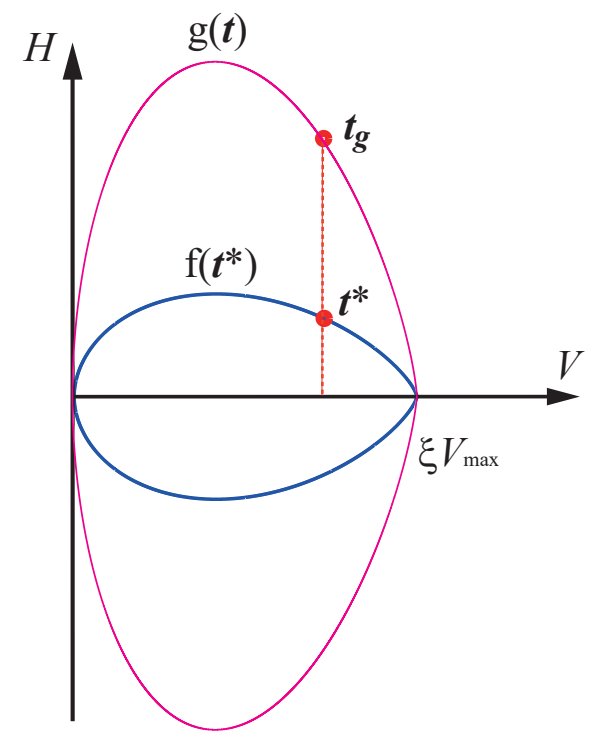

Figure 9: Current loading surface and plastic potential surface

${ }_{161}$ An image point $\boldsymbol{t}_{g}=\left[\begin{array}{ll}V_{g} & H_{g}\end{array}\right]^{T}$ can be easily found on the plastic potential surface with $V_{g}=V^{*}$ and 
162

$H_{g}=\frac{\lambda_{h}}{\lambda_{v}} H^{*}$. Then $\boldsymbol{m}$ can be determined by:

$$
\boldsymbol{m}=\frac{\partial g / \partial \boldsymbol{t}_{g}}{\left\|\partial g / \partial \boldsymbol{t}_{g}\right\|}
$$

In this study, before the model reaches full plasticity, i.e. $0<\xi<1, \lambda_{h}$ and $\lambda_{v}$ are constants. In order to avoid excessive plastic displacement of pipe invert (for example, the movement of pipe invert due to lateral displacement under constant vertical force), when fully plasticity is reached i.e. $\xi=1, \lambda_{v}$ is considered to be dependent on the lateral movement of the pipe. Therefore $\lambda_{v}$ is defined by eq. (16) as:

$$
\lambda_{v}= \begin{cases}1.0 & \text { if } 0<\xi<1 \\ 25 *(u / D)^{2} & \text { if } \xi=1\end{cases}
$$

where $u$ and $D$ are the lateral displacement and the pipe diameter respectively. According to Eq. (16), when the lateral displacement $u$ is sufficiently large, the development of plasticity turns gradually to the direction of the lateral movement of the pipe. Eq. (16) captures the effect that under certain vertical loads, pipes reach a steady embedment after undergoing a large lateral displacement $[5,7,9]$.

It should be pointed out that at the corners of the plastic potential function i.e. when the horizontal force is $H=0$, the function is not differentiable and therefore a special treatment is required. In this study, when $H=0$ the plastic flow direction is forced to be the same as the direction of the vertical displacement increment.

\section{Model validation}

In this section, model validation is carried out by comparing macroelement results with FEM simulations and experimental data. First of all the model parameters are summarized. Then the performance of the proposed macroelement is validated by probe penetration and sweep tests. Finally, the limits of the proposed macroelement are acknowledged and discussed.

\subsection{Model parameters}

For a soil-pipe system system with given pipe diameter $D$, embedment ratio $w / D$ and undrained shear strength $s_{\mathrm{u}}$, several model parameters can be directly calculated by equations presented in the above sections. The model parameters can be determined and calibrated by the following steps:

- Elastic stiffness: $k_{\mathrm{vv}}, k_{\mathrm{hh}}$ and $k_{\mathrm{hv}}$ can be calculated by Eqs. in Tabs. 1 and 2

- Ultimate bearing capacity envelope: $V_{\max }$ which determines the size of the ultimate bearing capacity envelope can be calculated by Eq. (10)

- Hardening and plasticity coupling parameter, $\kappa, \lambda_{\mathrm{h}}$ and $\lambda_{\mathrm{v}}$ can be determined by performing several monotonic simulations and comparing with the monotonic test data. Then the optimum values can be selected. The parameter $\kappa$ is responsible for the isotropic hardening of the plastic response which defines how fast the model state is approaching to the ultimate bearing capacity envelope. This parameter can be obtained by fitting the vertical penetration test data. (for example, the date of the initial vertical loading step in the test NO. 1.305.2 of Hodder and Cassidy [42]). Parameters $\lambda_{\mathrm{h}}$ and $\lambda_{\mathrm{v}}$, acting on the coupling effect of the development of plasticity between horizontal and vertical directions, can be calibrated by fitting the curve of horizontal sweep test data. (for example, the experimental data of test NO. 1.305.2 of Hodder and Cassidy [42] and data of Dingle et al. [49]).

- "Internal displacement": For other parameters such as the 5 parameters for the "internal displacement", they can be determined with a trial and error procedure using cyclic loading test data. For the newly proposed macroelement, these 5 parameters were empirically determined by matching the stiffness variation with the experimental cyclic test data with unloading and loading reversal paths. Thus, the two constants $m_{T}<=m_{R}$ can be determined from the fitting of the stiffness change when passing from the 
small-displacement to the medium-displacement regime and for full unloading path. The constant $R$, which provides the size of the elastic regime can be calibrated by trial and error procedure together with the calibration of $m_{T}$ and $m_{R} \cdot \chi$ and $\beta_{r}$ which are responsible for accumulation effects can be obtained by comparing with the experimental data with several cycles of loading. The proper values of $\chi$ and $\beta_{r}$ can capture the possible accumulation trend of deformation/force under cyclic loadings.

The main parameters for the proposed hypoplastic macroelement are summerized in Table. 5 .

Table 5: Model parameters of the proposed hypoplastic macroelement for a soil-pipe system

\begin{tabular}{|c|c|c|c|}
\hline Model parameter & Values & Description & Group \\
\hline$k_{\mathrm{vv}}$ & Eqs. in Tabs. 1 and 2 & Vertical stiffness & \multirow{3}{*}{ Elastic stiffness } \\
\hline$k_{\mathrm{hh}}$ & Eqs. in Tabs. 1 and 2 & Horizontal stiffness & \\
\hline$k_{\mathrm{hv}}$ & Eqs. in Tabs. 1 and 2 & Coupled stiffness & \\
\hline$V_{\max }$ & Eq. (10) & Limit bearing capacity & \multirow{2}{*}{ Bearing capacity envelope } \\
\hline$\alpha$ & 0 or 1 & Contact condition & \\
\hline$\kappa$ & 1.2 & Loading function constant & Hardening \\
\hline \multirow[t]{2}{*}{$\lambda_{\mathrm{h}}$} & 2.5 & For rough contact & \multirow{3}{*}{ Direction of plasticity evolution } \\
\hline & 1.5 & For frictionless contact & \\
\hline$\lambda_{\mathrm{v}}$ & Eq. (16) & For all cases & \\
\hline$m_{R}$ & 2.5 & Stiffness at load reversal point & \multirow{5}{*}{$\begin{array}{l}\text { Cyclic behavior } \\
\text { (internal displacement) }\end{array}$} \\
\hline$m_{T}$ & 2.0 & Stiffness when neutral loading & \\
\hline$R$ & $1.0 \times 10^{-3}$ & Range of linearity & \\
\hline$\beta_{r}$ & 0.1 & Rate of evolution of IS & \\
\hline$\chi$ & 0.2 & Transition of stiffness & \\
\hline
\end{tabular}

\subsection{Probe penetration and penetration-sweep tests}

In this section, the model validation is carried out for the probe penetration and penetration-sweep tests. This kind of tests can validate the evolution of pipe resistance paths within the ultimate bearing capacity envelope of the soil-pipe system. For the probe penetration tests, vertical and horizontal displacements were applied simultaneously at the center of the pipe. A displacement control method was used and the reaction forces were recorded. The elastic FEM model presented in section. 4.1 was used and new plastic material properties were assigned to refine the model response. The Mohr-Coulomb yield criterion which is an isotropic elasto-plastic constitutive model was adopted to model the soil plasticity. Undrained shear strength $s_{u}$ and $0^{\circ}$ of friction angle and dilatation angle were used as the model parameters. As mentioned in section. 2, a parameter $\alpha$ is used to define the contact condition ( 0 for frictionless and 1 for rough contact condition). In the plastic model, the shear strength of the contact interface between the pipe and the soil is $\tau_{\max }=\alpha s_{\mathrm{u}}$.

The different simulation cases are summarized in Table. 6 and comparisons of the macroelement and the FEM numerical simulations are shown in Figs. 10, 11 and 12. The results are shown in terms of normalized horizontal (or vertical) resistance and normalized embedment (or lateral movement). For different soil profiles, different contact conditions, pipe diameters etc., the prediction of the macroelelement model matches well with the numerical results. The initial branch of the curves indicates a good prediction of the stiffness. For the evolution of vertical and lateral resistances, the macroelement has also a satisfactory performance. It can be also noticed that the ultimate resistance develops rapidly even under relative small movement of the pipe. The peak value is found for a lateral movement less than $0.01 u / D$. 
Table 6: Loading cases for probe penetration test

\begin{tabular}{ccclccc}
\hline NO. & $D$ & $w / D$ & Modulus profile & $s_{\mathrm{u}}$ & Contact condition & Applied displacements \\
\hline 1 & $2.0 \mathrm{~m}$ & 0.4 & Constant $(E=200 \mathrm{MPa})^{200 \mathrm{kPa}}$ & rough & $w=0.2 \mathrm{~m}$ and $u=0.083 \mathrm{~m}$ \\
\hline 2 & $1.5 \mathrm{~m}$ & 0.3 & Linear $^{*}(E=z m)$ & $200 \mathrm{kPa}$ & rough & $w=0.3 \mathrm{~m}$ and $u=0.1 \mathrm{~m}$ \\
\hline 3 & $1.0 \mathrm{~m}$ & 0.5 & Constant $(E=200 \mathrm{MPa})$ & $300 \mathrm{kPa}$ & frictionless & $w=0.12 \mathrm{~m}$ and $u=0.04 \mathrm{~m}$ \\
\hline$z$ is depth $; m=200 \mathrm{MPa} / 6 \mathrm{~m}$ & & & &
\end{tabular}

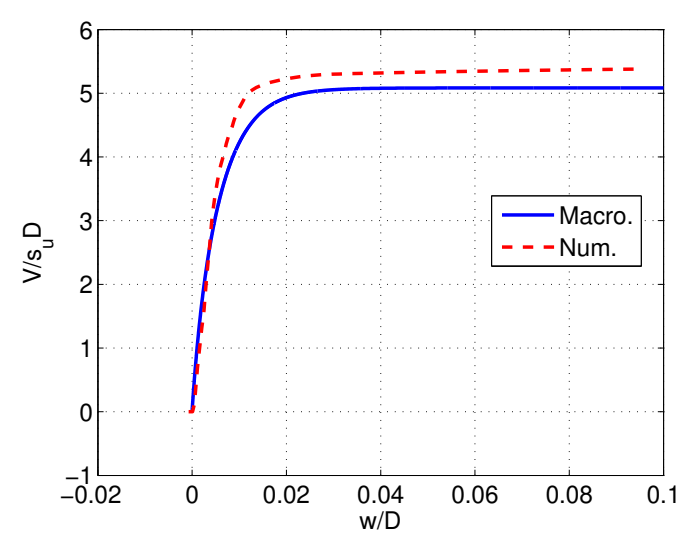

(a)

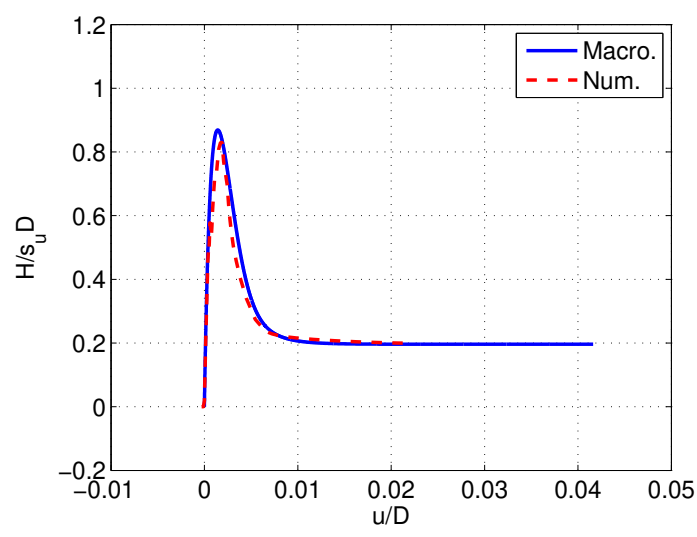

(b)

Figure 10: Validation of the proposed macroelement: $D=2 \mathrm{~m}$; constant modulus profile ( $E=200 \mathrm{MPa}) ; s_{\mathrm{u}}=200 \mathrm{kPa}$; rough contact condition; $w / D=0.4$. $w=0.2 \mathrm{~m}$ and $u=0.083 \mathrm{~m}$. (a) Vertical response (b) Horizontal response

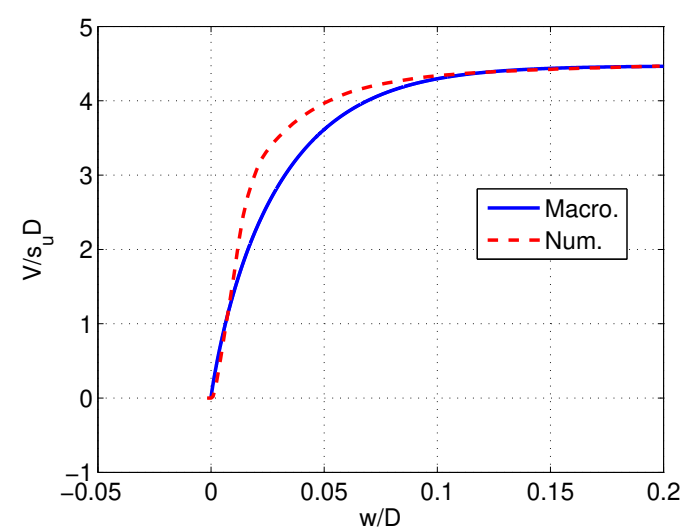

(a)

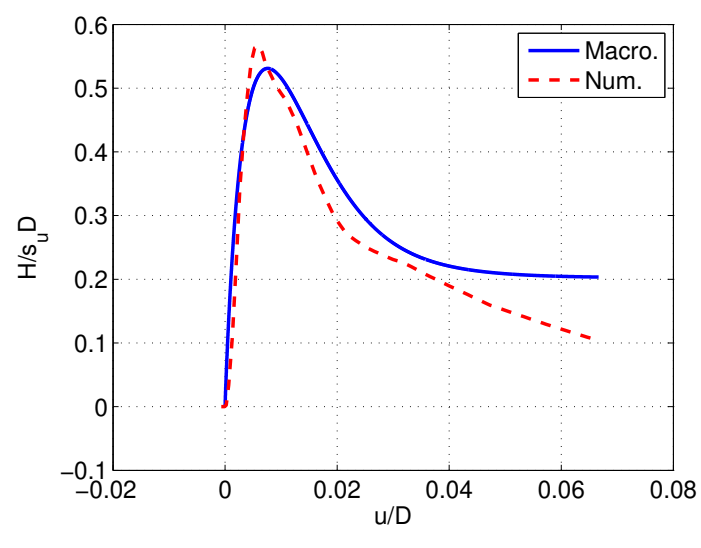

(b)

Figure 11: Validation of the proposed macroelement: $D=1.5 \mathrm{~m}$; linear modulus profile $(E=z m$, where $z$ is depth; $m=200 \mathrm{MPa} / 6 \mathrm{~m})$; $s_{\mathrm{u}}=200 \mathrm{kPa}$; rough contact condition; $w / D=0.3 . w=0.3 \mathrm{~m}$ and $u=0.1 \mathrm{~m}$ (a) Vertical response (b) Horizontal response

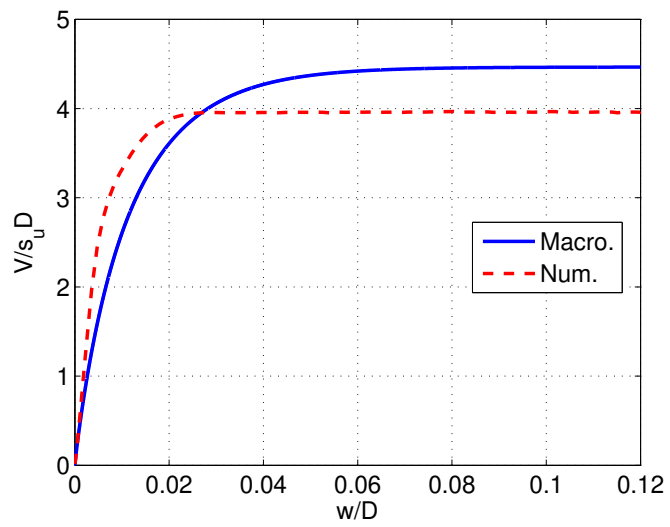

(a)

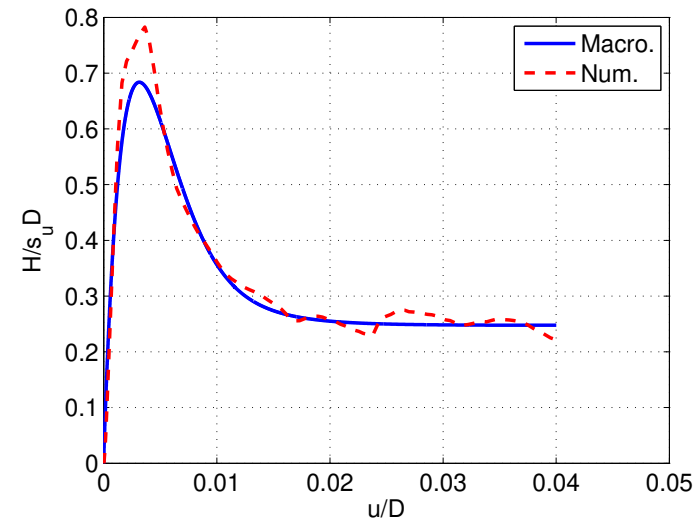

(b)

Figure 12: Validation of the proposed macroelement: $D=1.0 \mathrm{~m}$; constant modulus profile ( $E=200 \mathrm{MPa}) ; s_{\mathrm{u}}=300 \mathrm{kPa}$; frictionless contact condition; $w / D=0.5$. $w=0.12 \mathrm{~m}$ and $h=0.04 \mathrm{~m}$ (a) Vertical response (b) Horizontal response 
Besides the penetration test, penetration-sweep tests $[12,54-56]$ of the soil-pipe system were also carried out. A penetration test is displacement controlled and has two steps. First, the pipe is pushed in the vertical direction until reaching a prescribed embedment. Then it was displaced horizontally while maintaining the vertical displacement. Taking the first example of pipe diameter and soil properties in Table. 6 , two displacement loading paths were simulated:(1) $u_{\mathrm{v}}=0.015 \mathrm{~m}$ then $u_{\mathrm{h}}=0.01 \mathrm{~m}$; (2) $u_{\mathrm{v}}=0.005 \mathrm{~m}$ then $u_{\mathrm{h}}=0.01 \mathrm{~m}$. The comparison of the macroelement results with FEM simulations is shown in Fig. 13. In the normalized $\frac{V}{S_{u} D}, \frac{H}{S_{u} D}$ space, the macroelement model can well capture the evolution of the loading path within the ultimate bearing capacity envelope.

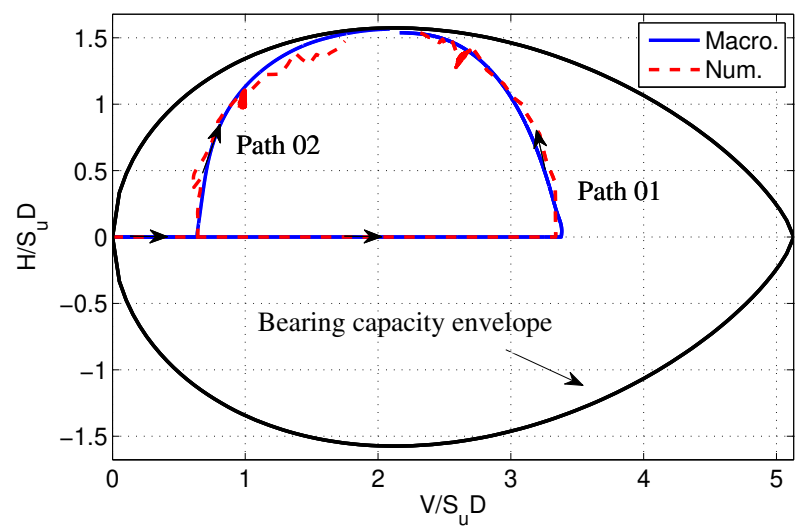

Figure 13: Validation of the proposed macroelement by swipe tests: $D=2.0 \mathrm{~m}$; constant modulus profile $(E=200 \mathrm{MPa}) ; s_{\mathrm{u}}=200 \mathrm{kPa}$; rough contact condition

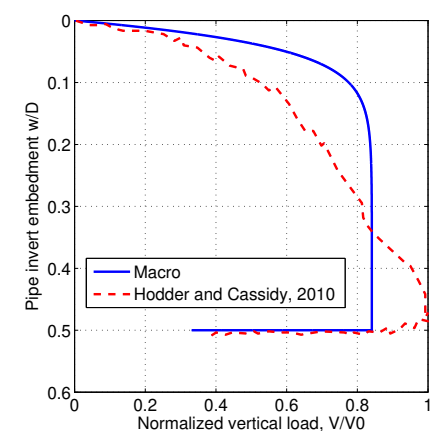

(a)

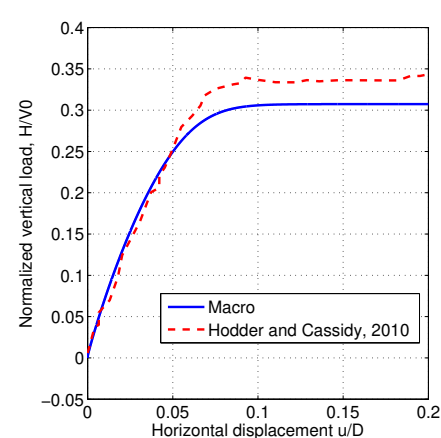

(b)

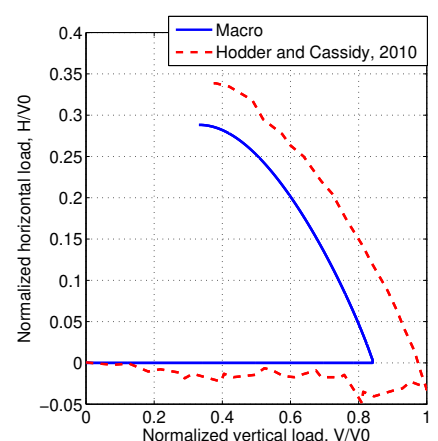

(c)

Figure 14: Comparison of macroelement simulations with the centrifuge test (Hodder and Cassidy, 2010), test NO. 1.305.2(a)[42]; $w / D=$ 0.5. (a) Vertical response (b) Horizontal response (c) Combined response

\section{5.3. Horizontal sweep test at constant vertical load}

In section 5.2, the validation of the macroelement is carried out by probe penetration and penetration-sweep tests. Generally, the ultimate resistance is fully developed at a relatively small displacement of pipe diameter. In this section, new series of lateral sweep tests are performed in which the pipe is subjected to relatively larger lateral displacement. Different from the sweep test in section 5.2, when the lateral sweep horizontal displacement is applied, instead of keeping a constant vertical displacement, a constant vertical force is maintained. This kind 


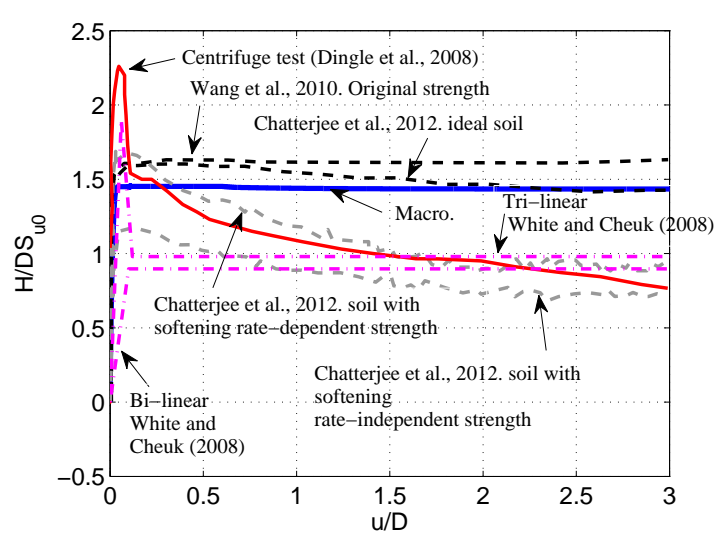

(a)

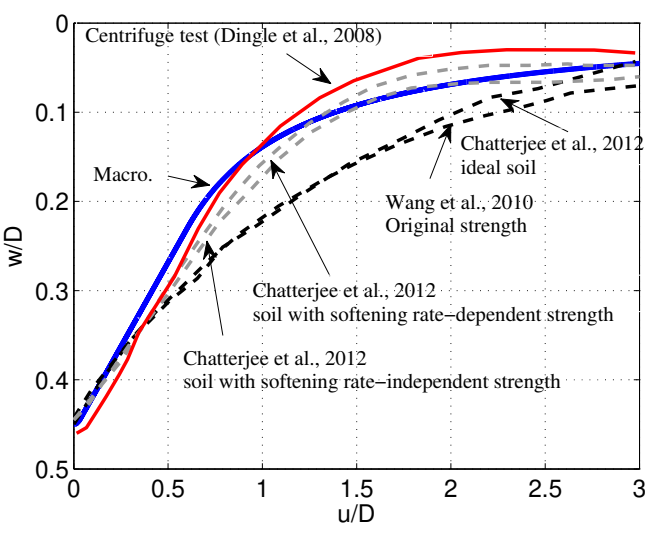

(b)

Figure 15: Comparison with experimental data (Wang et al., 2010 [5] and Chatterjee et al., 2012[6, 7]). Lateral displacement under constant vertical force; initial embedment $w / D=0.45$. (a) lateral resistance response (b) pipeline trajectory during lateral movement

of sweep test allows the vertical movement of pipe invert under pipe lateral displacement. First, the macroelement model is compared is compared with the centrifuge test performed by Dingle et al. [49], numerical results from Chatterjee et al. [6, 7], bilinear and tri-linear resistance models from White and Cheuk [57]. The pipe diameter $D$ is $0.8 \mathrm{~m}$ with an embedment ratio $w / D$ equals to 0.45 . The soil shear strength is $2.3 \mathrm{kPa}$ with a strength gradient $k$ equals to $3.6 \mathrm{kPa} / \mathrm{m}$. The Young's modulus of the soil is taken as $500 s_{u 0}\left(s_{u 0}=s_{u}+k z\right)$. A rough contact was assumed in the macroelement simulation. For the bi-linear and tri-linear models, related parameters mentioned above, the friction factor $\mu=0.5$ and the submerged unit weight $\gamma^{\prime}=6.5 \mathrm{kN} / \mathrm{m}^{3}$ are adopted to calculate the value of limiting horizontal force, breakout resistance and the constant residual force. The specific calculation equations can be found in the research of White and Cheuk [57].The values of $u_{\text {breakout }} / D=0.1$ and $u_{\text {residual }} / D=0.25$ to define the mobilization distances of these two models' response is recommended as typical values in their research. As a comparison, two key distances, i.e. $u_{\text {break }}$ and $u_{\text {residual }}$ from the centrifuge test of Dingle et al. [49] are also introduced into these two models. The comparison of the results is shown in Fig. 15. In Fig. 15(a), the centrifuge test result of Dingle et al. [49] and the empirical equation calculations of tri-linear model show a sudden breakout behaviour at the early stage of the lateral movement. This brittle behaviour is primarily due to the loss of suction at the rear of the pipe. The magnitude of the breakout resistance is not governed by the undrained shear strength $s_{u}$ but by the maximum available negative excess pore pressure and tensile resistance [49]. Neither the macroelement nor the FEM model can capture well this brittle behavior. Apart from the this phenomenon, the results of the macroelement are closer to the numerical results obtained negelcting softening and rate effects, see Fig. 15(a). Actually, since the macroelement model is based on the capacity envelope proposed by Merifield et al. [2] which is obtained numerically using a FEM model with an ideal soil strength (no softening and rate effects), it cannot take into account softening and rate-dependent effects. For the macroelement, the FEM model and the centrifuge test data, the horizontal resistance grows rapidly and reaches the maximum then the pipe undergoes very large lateral movement till 3 times the pipe diameter. It is also shown in Fig. 15(b) that the macroelement can capture the invert movement when lateral movement occurs under constant vertical load. This is due to the various couplings considered by the macroelement.

Fig. 16 presents the comparison of the macroelement with the FEM results of Chatterjee et al. [6, 7]. In this case, Chatterjee et al. [6,7] performed a parametric study of the influence of the vertical load on pipe invert movement under lateral displacement. The pipe diameter $D$ is $0.8 \mathrm{~m}$ and the embedment ratio $w / D$ equals 0.3 . The soil shear strength is $2.0 \mathrm{kPa}$ with a strength gradient $k$ equals to $4.0 \mathrm{kPa} / \mathrm{m}$. The Young's modulus of the soil is taken as $500 s_{u 0}\left(s_{u 0}=s_{u}+k z\right)$. The macroelement captures well the trend of pipe invert movement. The pipe invert finally reaches a steady embedment after the lateral displacement of 2 times of the pipe diameter. Another validation is carried out by comparing with the numerical results of Wang et al. [5] and summarized in Fig. 17. The pipe diameter $D$ is $0.8 \mathrm{~m}$ and the embedment ratio $w / D$ equals 0.45 . The soil shear strength is 2.3 
$\mathrm{kPa}$ with a strength gradient $k$ equals to $3.6 \mathrm{kPa} / \mathrm{m}$. As shown in Figs 16 and 17, the phenomenon where lighter pipes rise and heavier pipes move downwards is reproduced.

The perfermance of the macroelement model can be explained by the direction of plastic flow. As it is shown in Fig. 18, if the vertical load is in the range of light pipe region, the direction of plastic flow $\boldsymbol{m}$ has a component which in the opposite direction of the vertical force. Thus, under lateral displacement, due to the coupling effect, the pipe invert moves in the opposite direction of the vertical load. The pipe therefore rises up. The opposite is true for the case of heavy pipelines. For a medium heavy pipe $\left(V / V_{\max }\right.$ around 0.4$)$, the movement of pipe invert is limited.

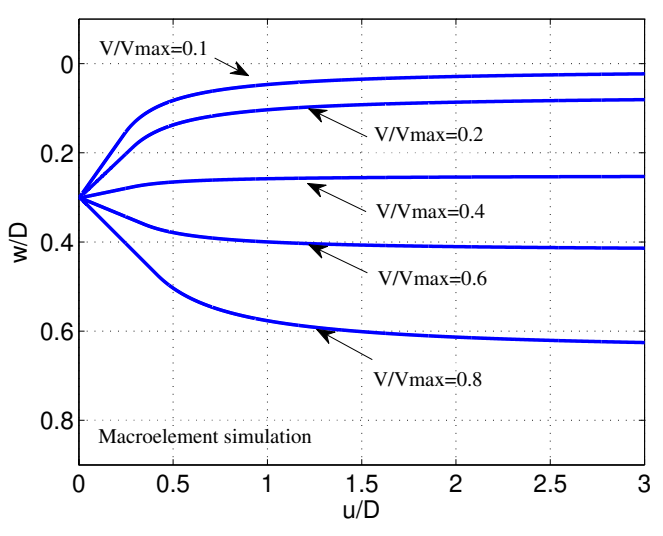

(a)

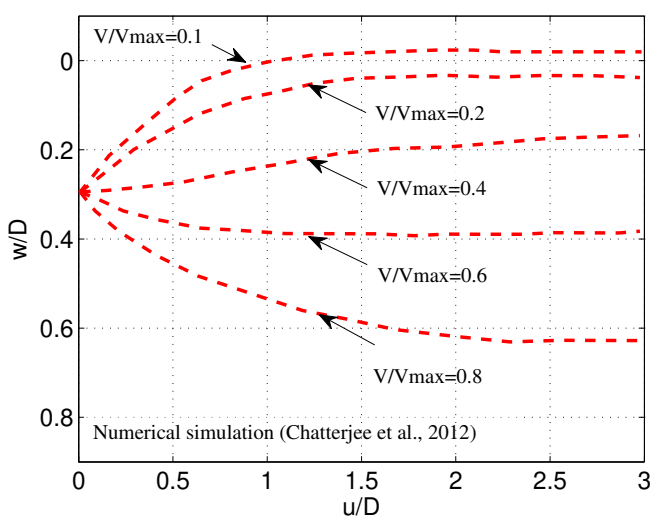

(b)

Figure 16: Validation of performance of proposed macroelement: lateral deformation under constant vertical force; initial embedment $w / D=0.3$. (a) Macroelement (b) Numerical simulation (Chatterjee et al.[6, 7])

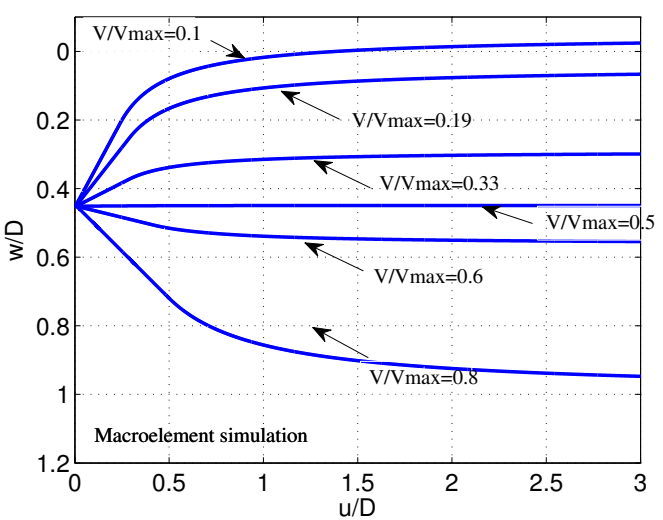

(a)

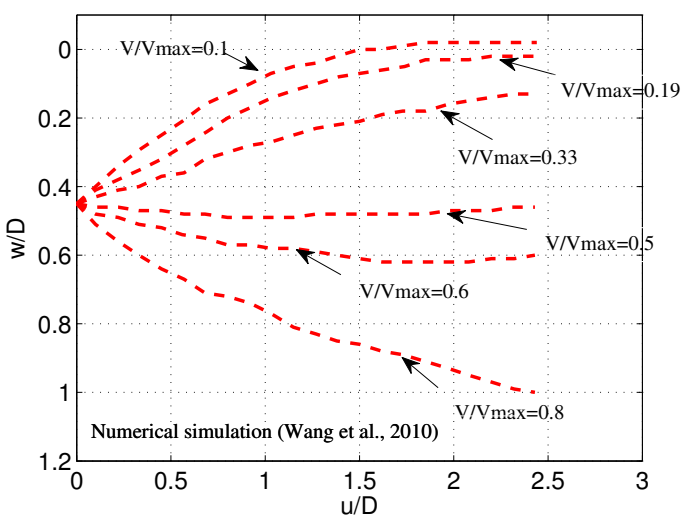

(b)

Figure 17: Validation of performance of proposed macroelement: lateral deformation under constant vertical force; initial embedment $w / D=0.45$. (a) Macroelement (b) Numerical simulation (Wang et al., 2010[5])

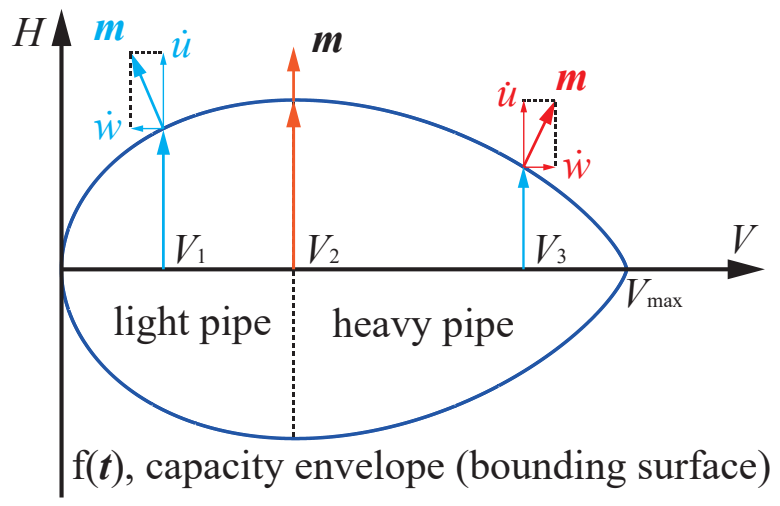

Figure 18: Development of plastic deformation in the case of light and heavy pipes 
macroelement. Centrifuge tests JIP3 and WA1 performed by Cheuk et al. [58] are used for the validation. JIP3 and WA1 were performed using E-grade kaolin clay and West African soft clay respectively. The total number of sweeps for JIP3 and WA1 are 6 and 14 cycles. Comparison is shown in Figs. 19, 20, 21 and 22. The macroelement can well predict the trend of the cyclic response except the degradation of the residual resistance with the increasing cyclic number. Under lateral cyclic movement, a significant increase of the horizontal resistance is observed due to the berm formation during horizontal loading. The soil berm formation mechanism cannot be captured with the proposed macroelement.

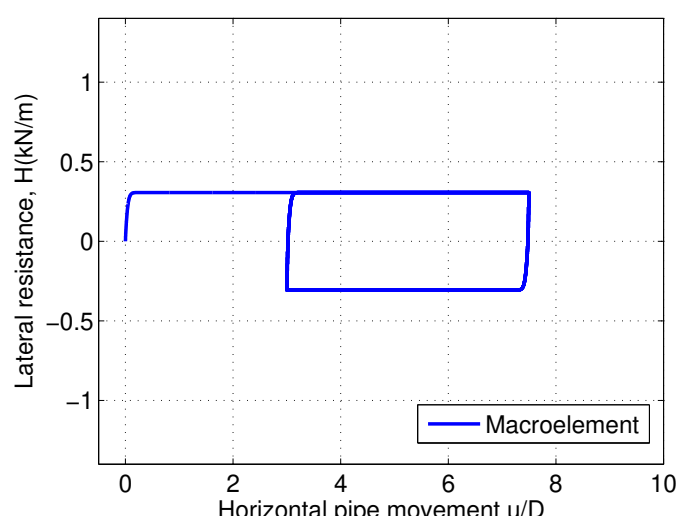

(a)

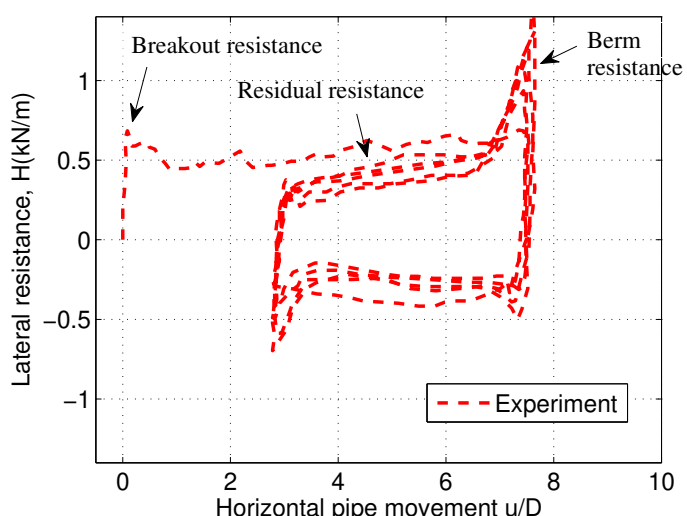

(b)

Figure 19: Validation of performance of proposed macroelement: horizontal resistance response. Initial embedment $w / D=0.088$. (a) Macroelement (b) Experimental data (Cheuk et al., 2007[58], test JIP3)

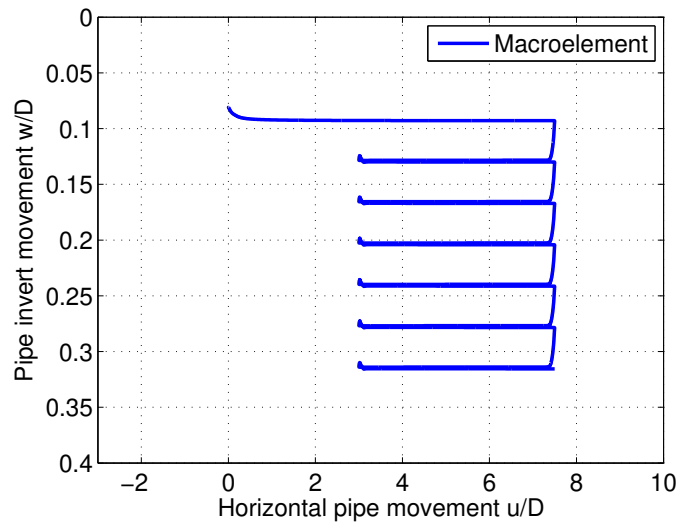

(a)

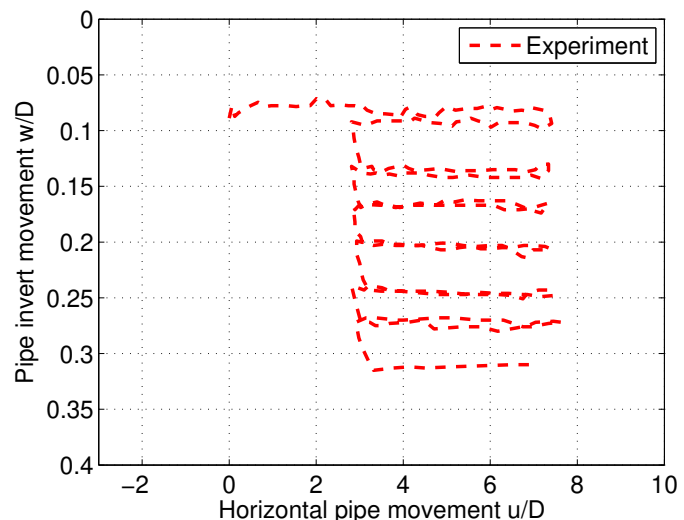

(b)

Figure 20: Validation of performance of proposed macroelement: pipe invert trajectory. Initial embedment $w / D=0.088$. (a) Macroelement (b) Experimental data (Cheuk et al., 2007[58], test JIP3)

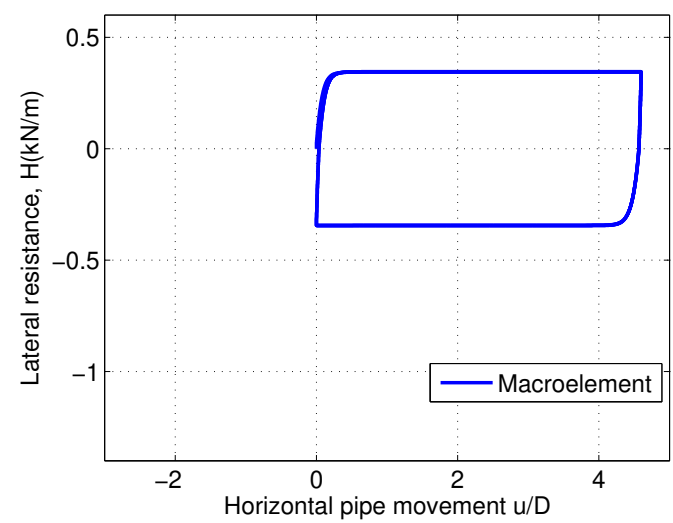

(a)

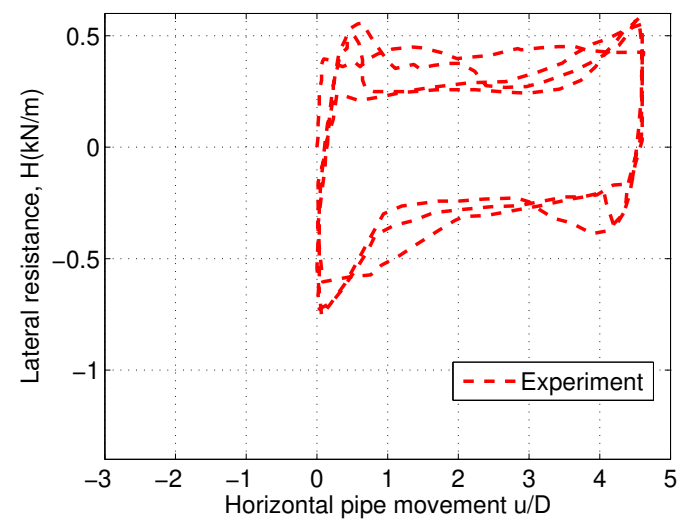

(b)

Figure 21: Validation of performance of proposed macroelement: horizontal resistance response. Initial embedment $w / D=0.557$. (a) Macroelement (b) Experimental data (Cheuk et al., 2007[58], test WA1) 


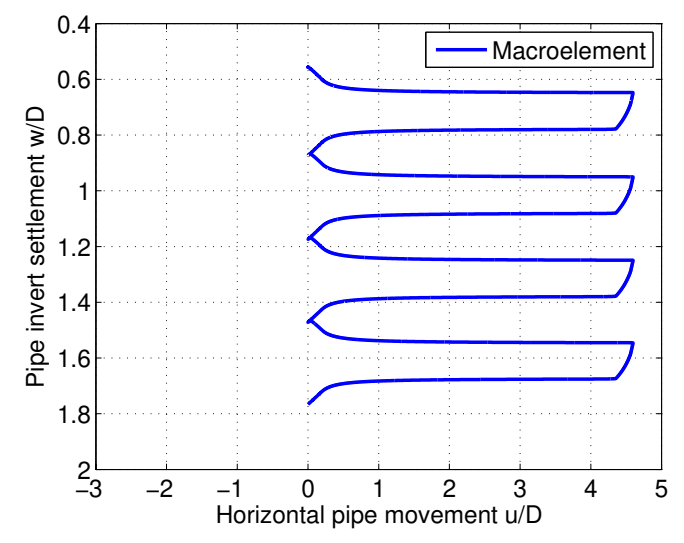

(a)

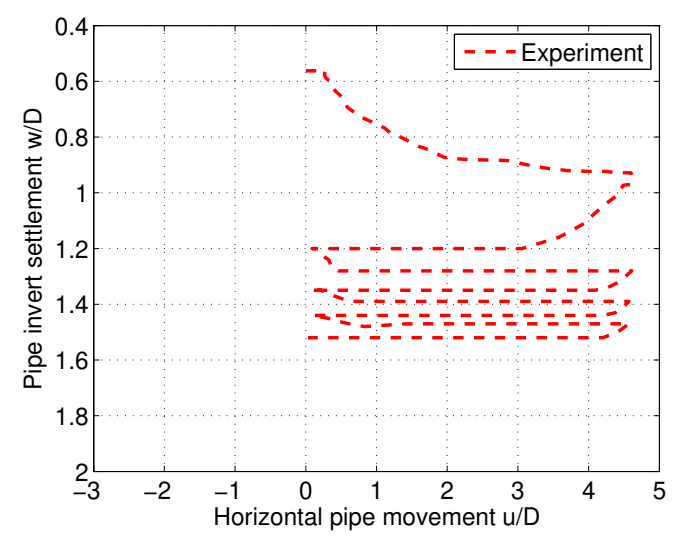

(b)

Figure 22: Validation of performance of proposed macroelement: pipe invert trajectory. Initial embedment $w / D=0.557$. (a) Macroelement (b) Experimental data (Cheuk et al., 2007[58], test WA1)

\section{Conclusions}

The modeling of pipe-soil interaction under complex loading conditions is an interesting and challenging

321 for her help. 


\section{References}

[1] D. Bruton, D. White, C. Cheuk, M. Bolton, M. Carr, Pipe/soil interaction behavior during lateral buckling, including large-amplitude cyclic displacement tests by the safebuck JIP, in: Offshore Technology Conference, Houston, Texas, USA, 2006, pp. 1-10. doi : 10.4043/17944-MS.

[2] R. Merifield, D. J. White, M. F. Randolph, The ultimate undrained resistance of partially embedded pipelines, Géotechnique 58 (6) (2008) 461-470. doi : 10.1680/geot. 2007.00097.

[3] Y. Tian, M. J. Cassidy, Modeling of Pipe-Soil Interaction and Its Application in Numerical Simulation, International Journal of Geomechanics 8 (4) (2008) 213-229. doi:10.1061/(ASCE) 1532-3641 (2008) 8.

[4] S. Chatterjee, D. J. White, M. F. Randolph, Coupled consolidation analysis of pipe - soil interactions, Canadian Geotechnical Journal 50 (6) (2013) 609-619. doi : $10.1139 / c g j-2012-0307$.

[5] D. Wang, D. J. White, M. F. Randolph, Large-deformation finite element analysis of pipe penetration and large-amplitude lateral displacement, Canadian Geotechnical Journal 47 (8) (2010) 842-856. doi: 10.1139/T09-147.

[6] S. Chatterjee, D. White, M. Randolph, Numerical simulations of pipe - soil interaction during large lateral movements on clay, Géotechnique 62 (8) (2012) 693-705. doi:10.1680/geot.10.P.107.

[7] S. Chatterjee, Numerical modelling of pipe-soil interactions, Phd thesis, University of Western Australia (2012).

[8] S. Chatterjee, M. Randolph, D. White, The effects of penetration rate and strain softening on the vertical penetration resistance of seabed pipelines, Géotechnique 62 (7) (2012) 573-582. doi:10.1680/geot. 10.P. 075 .

[9] S. Chatterjee, D. White, M. Randolph, Numerical simulations of pipe - soil interaction during large lateral movements on clay, Géotechnique 62 (8) (2012) 693-705. doi :10.1680/geot.10.P.107.

[10] S. Dutta, B. Hawlader, R. Phillips, Finite element modeling of partially embedded pipelines in clay seabed using Coupled Eulerian - Lagrangian method, Canadian Geotechnical Journal 52 (1) (2014) 58-72. doi : doi.org/10.1139/cgj-2014-0045.

[11] R. Nova, L. Montrasio, Settlements of shallow foundations on sand, Géotechnique 41 (2) (1991) $243-256$.

[12] G. Gottardi, G. Houlsby, R. Butterfield, Plastic response of circular footings on sand under general planar loading, Géotechnique 49 (4) (1999) 453-470.

[13] L. Montrasio, R. Nova, Settlements of shallow foundations on sand: geometrical effects, Géotechnique 47 (1) (1997) 49-60.

[14] R. Paolucci, Simplified Evaluation of Earthquake-Induced Permanent Displacements of Shallow Foundations, Journal of Earthquake Engineering 1 (3) (1997) 563-579. doi : 10. 1080/13632469708962378.

[15] C. Cremer, A. Pecker, L. Davenne, Cyclic macro-element for soil-structure interaction: Material and geometrical non-linearities, International Journal for Numerical and Analytical Methods in Geomechanics 25 (13) (2001) 1257-1284. doi:10.1002/nag. 175.

[16] Y. Le Pape, J. Sieffert, Application of thermodynamics to the global modelling of shallow foundations on frictional material, International Journal for Numerical and Analytical Methods in Geomechanics 25 (2001) 1377-1408. doi:10.1002/nag.186. 
[17] C. Martin, G. Houlsby, Combined loading of spudcan foundations on clay: numerical modelling, Géotechnique 51 (8) (2001) 687-699.

[18] M. Cassidy, B. Byrne, G. Houlsby, Modelling the behaviour of circular footings under combined loading on loose carbonate sand, Géotechnique 52 (10) (2002) 705-712.

[19] C. Cremer, A. Pecker, L. Davenne, Modelling of nonlinear dynamic behaviour of a shallow strip foundation with macro-element, Journal of Earthquake Engineering 6 (2) (2002) 175-211.

[20] S. Grange, P. Kotronis, J. Mazars, A macro-element to simulate 3D soil-structure interaction considering plasticity and uplift, International Journal of Solids and Structures 46 (20) (2009) 3651-3663. doi: 10.1002/nag. 664 .

[21] C. T. Chatzigogos, R. Figini, A. Pecker, J. Salençon, A macroelement formulation for shallow foundations on cohesive and frictional soils, International Journal for Numerical and Analytical Methods in Geomechanics 35 (8) (2011) 902-931. doi:10.1002/nag. 934.

[22] B. Bienen, B. W. Byrne, G. T. Houlsby, M. J. Cassidy, Investigating six-degree-of-freedom loading of shallow foundations on sand, Géotechnique 56 (6) (2006) 367-379.

[23] M. J. Cassidy, C. M. Martin, G. T. Houlsby, Development and application of force resultant models describing jack-up foundation behaviour, Marine Structures 17 (2004) 165-193. doi:10.1016/j. marstruc. 2004.08.002.

[24] C. Prisco, A. Galli, Soil-pipe interaction under monotonic and cyclic loads : experimental and numerical modelling, in: Proceedings of the First Euro Mediterranean Symposium in Advances on Geomaterials and Structures, 2006, pp. 755-760.

[25] G. Cocchetti, C. di Prisco, A. Galli, Soil-pipeline interaction along unstable slopes: A coupled threedimensional approach. Part 2: Numerical analyses, Canadian Geotechnical Journal 46 (11) (2009) 13051321. doi:10.1139/T09-102.

[26] N. Zamani, U. El Shamy, Analysis of the seismic response of soil-foundation-structure systems using a microscale framework, Soil Dynamics and Earthquake Engineering 43 (2012) 398-412. doi:10.1016/ j.soildyn.2012.07.010.

URL http://dx.doi.org/10.1016/j.soildyn.2012.07.010

[27] G. Cocchetti, C. di Prisco, A. Galli, R. Nova, Soil-pipeline interaction along unstable slopes: a coupled three-dimensional approach. Part 1: Theoretical formulation, Canadian Geotechnical Journal 46 (11) (2009) 1289-1304. doi:10.1139/T09-028.

[28] S. hyeon Chai, A. R. Ghaemmaghami, O. S. Kwon, Numerical modelling method for inelastic and frequency-dependent behavior of shallow foundations, Soil Dynamics and Earthquake Engineering 92 (August 2016) (2017) 377-387. doi:10.1016/j . soildyn.2016 .10.030.

[29] I. Anastasopoulos, R. Kourkoulis, F. Gelagoti, E. Papadopoulos, Rocking response of SDOF systems on shallow improved sand: An experimental study, Soil Dynamics and Earthquake Engineering 40 (2012) 15-33. doi:10.1016/j.soildyn.2012.04.006.

[30] D. Kolymbas, An outline of hypoplasticity, Archive of applied mechanics 61 (3) (1991) 143-151.

[31] C. Tamagnini, G. Viggiani, R. Chambon, A review of two different approaches to hypoplasticity, Constitutive modelling of granular materials, in: Constitutive modelling of granular materials, Springer, Berlin, Heidelberg, 2000, pp. 107-145. 
[32] A. Niemunis, Extended hypoplastic models for soils, Habilitation Thesis, Inst. für Grundbau und Bodenmechanik (2003).

[33] D. Salciarini, C. Tamagnini, A hypoplastic macroelement model for shallow foundations under monotonic and cyclic loads, Acta Geotechnica 4 (3) (2009) 163-176. doi:10.1007/s11440-009-0087-2.

[34] C. Tamagnini, D. Salciarini, R. Ragni, Implementation of a 6-dof hypoplastic macroelement in a finite element code, in: COM. Geo 2012: proceedings of the 3rd international conference on computing for geospatial research and applications, Washington, DC, USA, 2013, pp. 60-71.

[35] Z. Jin, Z.-y. Yin, P. Kotronis, Z. Li, C. Tamagnini, A hypoplastic macroelement model for a caisson foundation in sand under monotonic and cyclic loadings, Marine Structures 66 (2019) 16-26. doi:10. 1016/j.marstruc.2019.02.002.

[36] Z. Li, P. Kotronis, S. Escoffier, C. Tamagnini, A hypoplastic macroelement for single vertical piles in sand subject to three-dimensional loading conditions, Acta Geotechnica 11 (2) (2016) 373-390. doi: $10.1007 / \mathrm{s} 11440-015-0415-7$.

[37] Z. Li, P. Kotronis, S. Escoffier, C. Tamagnini, A hypoplastic macroelement formulation for single batter piles in sand, International Journal for Numerical and Analytical Methods in Geomechanics 42 (12) (2018) 1346-1365. doi:10.1002/nag. 2794.

[38] G. Schotman, F. Stork, Pipe-soil interaction: a model for laterally loaded pipelines in clay, in: Offshore Technology Conference, Houston, Texas, 1987, pp. 1-8.

[39] J. Zhang, D. Stewart, M. Randolph, Kinematic hardening model for pipeline-soil interaction under various loading conditions, The International Journal of Geomechanics 2 (4) (2002) 419-446.

[40] J. Zhang, D. P. Stewart, M. F. Randolph, Modeling of Shallowly Embedded Offshore Pipelines in Calcareous Sand, Journal of Geotechnical and Geoenvironmental Engineering 128 (5) (2002) 363-371. doi:10.1061/(ASCE) 1090-0241(2002) 128:5(363).

[41] M. F. Randolph, D. J. White, Upper-bound yield envelopes for pipelines at shallow embedment in clay, Géotechnique 58 (4) (2008) 297-301. doi:10.1680/geot.2008.58.4.297.

[42] M. S. Hodder, M. J. Cassidy, A plasticity model for predicting the vertical and lateral behaviour of pipelines in clay soils, Géotechnique 60 (4) (2010) 247-263. doi:10.1680/geot.8.P. 055.

[43] O. Hededal, T. Strandgaard, A 3D elasto-plastic spring element for pipe-soil interaction analysis, in: Offshore Pipeline Technology Conference, Amsterdam, 2008, pp. 1-8.

[44] Y. Tian, M. Cassidy, Pipe-soil interaction analysis with a three-dimensional macroelement model, in: The Nineteenth International Offshore and Polar Engineering Conference, Perth, Australia, 2009, pp. 1-8.

[45] A. Niemunis, I. Herle, Hypoplastic model for cohesionless soils with elastic strain range, Mechanics of Cohesive-frictional Materials 2 (1997) 279-299. doi:10.1002/(SICI) 1099-1484(199710)2: 4<279: : AID-CFM29>3.0. CO;2-8.

[46] M. J. Pender, D. P. Carter, S. Pranjoto, M. John, D. P. Carter, S. Pranjoto, M. J. Pender, Diameter Effects on Pile Head Lateral Stiffness and Site Investigation Requirements for Pile Foundation Design, Journal of Earthquake Engineering 11 (Sup1) (2007) 1-12. doi:10.1080/13632460701280005.

[47] S. Gajan, T. C. Hutchinson, B. L. Kutter, P. Raychowdhury, J. A. Ugalde, J. P. Stewart, Numerical Models for Analysis and Performance-Based Design of Shallow Foundations Subjected to Seismic Loading, Tech. rep., Pacific Earthquake Engineering Research Center (2008). 
[48] A. Giannakou, N. Gerolymos, G. Gazetas, T. Tazoh, I. Anastasopoulos, Seismic behavior of batter piles: elastic response, Journal of Geotechnical and Geoenvironmental Engineering 136 (9) (2010) 1187-1199.

${ }_{443}$ [49] H. R. C. Dingle, D. J. White, C. Gaudin, Mechanisms of pipe embedment and lateral breakout on soft clay, Canadian Geotechnical Journal 45 (5) (2008) 636-652. doi :10.1139/T08-009.

[50] C. Y. Cheuk, D. J. White, Modelling the dynamic embedment of seabed pipelines, Géotechnique 61 (1) (2011) 39-57. doi:10.1680/geot.8.P.148.

447 [51] D. J. White, H. R. C. Dingle, The mechanism of steady friction between seabed pipelines and clay soils, Géotechnique 61 (12) (2011) 1035-1041.

457 [55] C. M. Martin, G. T. Houlsby, Combined loading of spudcan foundations on clay: laboratory tests, Geotéchnique 50 (4) (2000) 325-338.

459 [56] M. F. Bransby, M. F. Randolph, Combined loading of skirted foundations, Geotéchnique 48 (5) (1998) $637-655$.

461 [57] D. J. White, C. Y. Cheuk, Modelling the soil resistance on seabed pipelines during large cycles of lateral movement, Marine Structures 21 (1) (2008) 59-79. doi :10.1016/j .marstruc.2007.05.001.

[58] C. Y. Cheuk, D. J. White, M. D. Bolton, Large-scale modelling of soil - pipe interaction during large amplitude cyclic movements of partially embedded pipelines, Canadian Geotechnical Journal 44 (8) (2007) 